\title{
High-fidelity simulations of electrolyte jets in an electric field
}

\author{
Venkata Krisshna*1, Mark Owkes ${ }^{2}$ \\ 1, 2 Department of Mechanical and Industrial Engineering, Montana State University, \\ Bozeman, USA \\ ${ }^{*}$ Corresponding author email: venkat.krisshna@gmail.com
}

\begin{abstract}
When an electrolyte jet is injected through a grounded nozzle into a region with an electric field, non-axisymmetric whipping instabilities are often observed in the jet. These instabilities are characterized by large scale violent, chaotic and quick whips of the jet. This system is numerically modeled using an electrohydrodynamic formulation that includes the Nernst-Planck model for ion transport with an aim to investigate the origin and propagation of the instabilities in the jet. Simulating this process will help gain an in-depth insight into the complex physical phenomena that occur. In this article, the formulation and modeling of electrolyte jets using a Poisson-Nernst-Planck (PNP) model is discussed.
\end{abstract}

\section{Keywords}

CFD, multiphase flow, whipping instabilities, electric field, NGA

\section{Introduction}

The Poisson-Nernst-Planck (PNP) model is extensively used to describe the electrodiffusion process of ions in electrolyte solutions. Modulation of liquid jets using a background electric field is used in several industrial processes such as electrospinning, electrospraying and ink-jet printing $[1,2,3]$. Several research groups have investigated instability dynamics of electrified liquid jets in background electric fields both analytically and numerically [4, 5, 6, 7, 8, 9]. However, these groups have studied the instabilities on a monopolarly charged, i.e., strictly positively or negatively charged liquid jets.

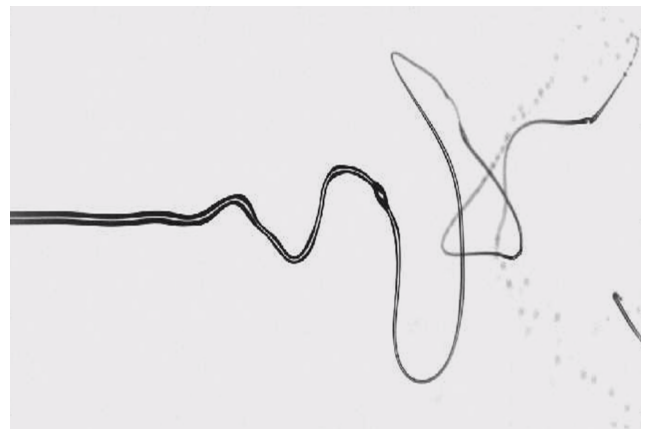

(a) Snapshot of experimentally observed instabilities. Image reproduced with permission from Multiphase \& Cardiovascular Flow Lab, University of Washington.

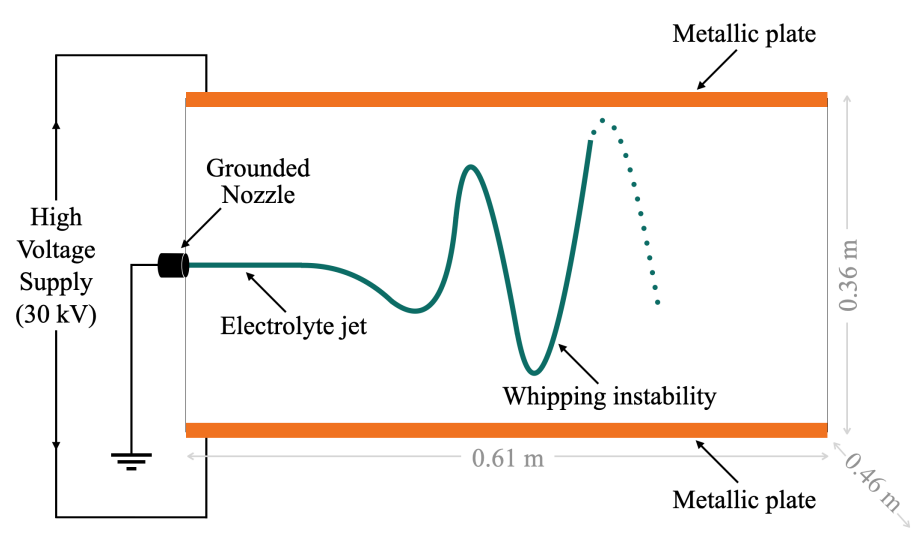

(b) Schematic of the experimental setup, not to scale

Figure 1. Experimentally observed whipping instabilities along an electrolyte jet in an electric field

In an experimental study performed at the Multiphase \& Cardiovascular Flow Laboratory (MCFL) of the University of Washington, sodium chloride solution of $55 \mathrm{ppm}$ concentration was injected at $\operatorname{Re} 1800$ through a grounded nozzle. The jet flows through a region of dimensions 35.56 $\times 45.72 \times 60.96 \mathrm{~cm}$ between two large metallic plates. A potential difference of $30 \mathrm{kV}$ exists between the grounded nozzle and the metallic plates. A snapshot of the observed trajectory and behavior of the jet is shown in Fig. 1a. A schematic of the experimental setup is shown in 
Fig. 1b. Of particular interest is the development and propagation of whipping instabilities along the jet. While such instabilities have been previously studied, they have been on monopolarly charged liquid jets $[10,11,12,13,14,15]$. The physics behind electrolyte jet dynamics in an electric field has not been explored. Such a jet comprises of an electrolytic solution, i.e., a solution of an electrolyte in a polar solvent (typically water). The electrolyte, once dissolved in the solution, ionizes into cations (positively charged) and anions (negatively charged) which disperse uniformly throughout the solvent thus rendering an electrically neutral solution. When a background electric field is applied, the ionic charges relax through a process called electromigration. The presence of ions carrying opposite charges in the same bulk liquid gives rise to more electrohydrodynamic (EHD) processes than in a monopolarly charged jet (which contains only like charges). We aim to characterize the physical processes that are observed in bipolarly charged (which contain both positive and negative charges) electrolyte jets in an electric field. Experimental results collected by the MCFL serve as useful data to 1) verify a numerical model for electrosprays and 2) understand the underlying physics that give rise to whipping instabilities along the electrolyte jet. Additionally, a multiphase PNP model can describe fluid transport in a variety of fields such as biophysics [16], electrochemistry [17], nanofluidics [18] and solid-state physics [19].

\section{Governing equations and methods}

The methods discussed below have been implemented within a code called NGA - a high-order, fully conservative, variable density, low Mach number Navier-Stokes solver that consists of various multi-physics modules implemented in parallel using message passing interface (MPI). The formulation discretely conserves mass and momentum in a periodic domain. NGA uses a conservative unsplit geometric volume-of-fluid (VOF) scheme. NGA has been developed by several groups to solve multiphase [20, 21, 22, 23, 24] and EHD flows [25, 26, 27]. In this project, we modify the existing EHD formulation to account for bipolar charges.

\section{Electrohydrodynamics}

The Maxwell stress tensor $\sigma_{i}^{\mathrm{e}}$ in Eq. 2 from [27] is given by

$$
\boldsymbol{\sigma}_{i}^{\mathrm{e}}=\varepsilon_{i} \mathbf{E}_{i} \otimes \mathbf{E}_{i}-\frac{\varepsilon_{i}}{2} \mathbf{E}_{i} \cdot \mathbf{E}_{i}\left(1-\frac{\rho_{i}}{\varepsilon_{i}} \frac{\partial \epsilon_{i}}{\partial \rho_{i}}\right) \mathbb{I}
$$

where $\varepsilon$ is the electric permittivity and $\mathbf{E}$ is the electric field vector. Magnetic effects have been ignored since the EHD time scale is several orders of magnitude larger than the magnetic time scale [28] in the process that is of interest here. This electrostatic assumption eliminates the effect of velocity of the charges (i.e., current) on the electric field thus dictating that the electric field is only influenced by the instantaneous electric charge distribution.

In the late 1800s, Nernst [29] and Planck [30] first provided a mathematical description for the process of electrodiffusion. Several groups have since developed numerical formulations of the PNP model [31, 32, 33, 34]. To accurately model ionization of charged species in electrolytes, we allow for separate treatment of cation $\left(c^{+}\right)$and anion $\left(c^{-}\right)$concentration fields. Electric forces $\mathbf{f}_{\text {electric }}$ can be expressed as the divergence of the Maxwell's stress tensor,

$$
\mathbf{f}_{\text {electric }}=\nabla \cdot \boldsymbol{\sigma}_{i}^{\mathrm{e}}=c^{ \pm} z^{ \pm} e \mathbf{E}_{i}-\frac{1}{2} \mathbf{E}_{i}^{2} \nabla \varepsilon_{i}+\nabla\left(\frac{1}{2} \rho_{i} \frac{\partial \varepsilon_{i}}{\partial \rho_{i}} \mathbf{E}_{i}^{2}\right),
$$

where $z^{+}$and $z^{-}$are the ionic valences associated with cationic and anionic charges respectively, and for a monovalent salt $z^{+}=-z^{-}=1$. $e$ is the elementary charge. The first term on the right hand side of Eq. 2 is the Coulomb (or Lorentz) force. The second and third terms denote the dielectric and the electrostrictive forces respectively and are only significant in a transient electric field with time scales several orders of magnitude larger than what is encountered in problems of our interest [35]. The electric field vector, which is irrotational due to the 
electrostatic assumption, can be expressed as the gradient of the electric potential $\phi$,

$$
\mathbf{E}_{i}=-\nabla \phi_{i}
$$

The electric potential Poisson equation is formulated as

$$
-\nabla \cdot\left(\varepsilon_{i} \nabla \phi_{i}\right)=z^{ \pm} e\left(c^{+}-c^{-}\right) .
$$

The PNP system governs the motion of ions due to i) convection in the velocity field $\mathbf{u}_{i}$ (advection), ii) electrostatic potential gradient (electromigration) and iii) chemical potential gradients (diffusion) while maintaining a self-consistent electric field. The ion transport equation is

$$
\frac{\partial c^{ \pm}}{\partial t}+\nabla \cdot\left(c^{ \pm} \mathbf{u}_{i}+\mathbf{N}^{ \pm}\right)=0
$$

where the molar flux $\mathbf{N}^{ \pm}$is formulated using the Nernst-Planck equation as

$$
\mathbf{N}^{ \pm}=D \frac{z^{ \pm} e}{k_{\mathrm{B}} T} c^{ \pm} \nabla \phi-D \nabla c^{ \pm}
$$

where $D^{ \pm}$is the diffusion constant of the charged species, $k_{\mathrm{B}}$ is the Boltzmann constant and $T$ is the ambient temperature. The two terms that contribute to the molar flux can be described as electromigration (advection due to the electrical velocity $D \frac{z^{ \pm} e}{k_{\mathrm{B}} T} \nabla \phi$ ) and diffusion.

In summary, ion concentration $c^{ \pm}$and boundary conditions (BCs) in $\phi$ are used to obtain $\phi$ using Eq. 4. $\mathbf{E}_{i}$ is then calculated using Eq. 3 after which Eq. 2 allows for the calculation of $\mathbf{f}_{\text {electric }}$ on the fluid element. Charges are transported as dictated by Eq. 5.

\section{Using e-Mesh}

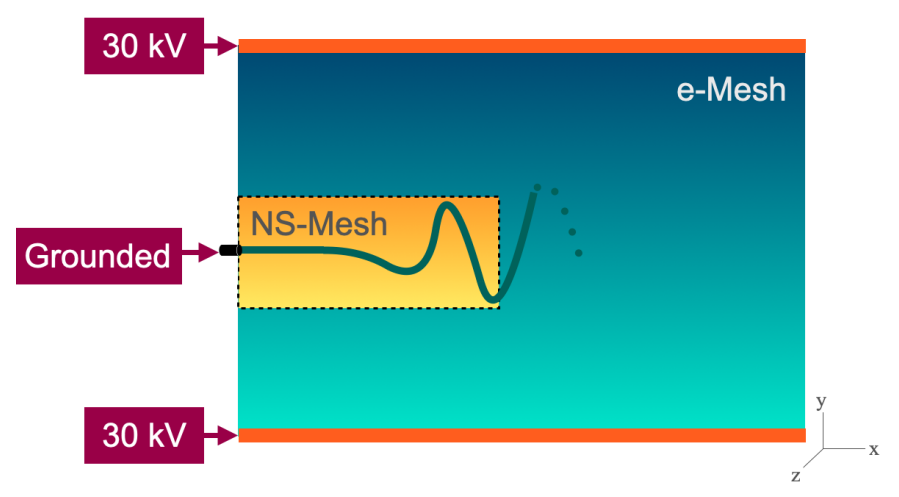

Figure 2. e-Mesh shown in context with NS-Mesh and available $\phi$ BCs

An addition to the EHD module of NGA developed for this project involves using a domain called e-Mesh. e-Mesh is much larger than the computational domain (henceforth called the NS-Mesh) where the fluid dynamics is solved. Since our interest lies in the development and propagation of whipping instabilities along the electrolyte jet, NS-Mesh occupies a small region near the liquid inflow. NS-Mesh requires a well-defined $\phi$ field in order to solve Eq. 3 and obtain the electric field. The $\phi$ field is obtained by solving Eq. 4 with appropriate BCs. However, $\phi$ values are not readily available at the walls of NS-Mesh. $\phi$ is well defined only at the inflow (grounded) and at the metallic plates $(30 \mathrm{kV})$. Instead of making an assumption at the walls of NS-Mesh, a new domain called e-Mesh that spans between regions of well-defined $\phi$ is initialized to obtain accurate BCs on NS-Mesh (Fig. 2). Neumann boundary conditions are imposed on the remaining walls of e-Mesh. To make NS-Mesh as large as e-Mesh would 
demand computational resources to solve fluid dynamics far from the jet which is not of interest in this project. Thus e-Mesh is used exclusively as an electric potential solver. In addition to the BCs, e-Mesh is populated with the $c^{ \pm}$and these are used to solve for $\phi$ on e-Mesh. Values of $\phi$ are interpolated from e-Mesh (which has a stretched grid and is coarser than NS-Mesh) to cells that lie on the walls of NS-Mesh using a tri-cubic interpolation method. These interpolated values are then used as BCs to compute $\phi$ on NS-Mesh.

\section{Results and discussion}

As discussed previously, there are two meshes involved in the methodology. The dimensions of e-Mesh will be $35.56 \times 45.72 \times 60.96 \mathrm{~cm}$, which is the size of the domain in the experimental setup. Dimensions of NS-Mesh will be determined as more simulations are performed. Before simulating liquid jets, efforts towards validation of the current PNP model are taken. The general simulation parameters for these tests are listed in Table 1.

Table 1. Values of simulations parameters for qualitative tests, unless otherwise specified

\begin{tabular}{c|c|c}
\hline Property & Value & Unit \\
\hline Liquid density & 1000 & $\mathrm{~kg} / \mathrm{m}^{3}$ \\
Gas density & 1 & $\mathrm{~kg} / \mathrm{m}^{3}$ \\
Liquid viscosity & $8.9 \times 10^{-4}$ & $\mathrm{~Pa} . \mathrm{s}$ \\
Gas viscosity & $1.8 \times 10^{-5}$ & $\mathrm{Pa.s}$ \\
Surface tension & $72.8 \times 10^{-3}$ & $\mathrm{~N} / \mathrm{m}$ \\
Length of domain & 2 & $\mathrm{~m}$ \\
Potential drop & 20 & $\mathrm{~V}$ \\
Liquid relative permittivity & 50 & - \\
Gas relative permittivity & 1 & - \\
Droplet diameter & 0.4 & $\mathrm{~m}$ \\
Diffusion coefficient & $1.6 \times 10^{-6}$ & $\mathrm{~m}^{2} / \mathrm{s}$ \\
lonic valence & 1 & - \\
Temperature & 300 & $\mathrm{~K}$ \\
\hline
\end{tabular}

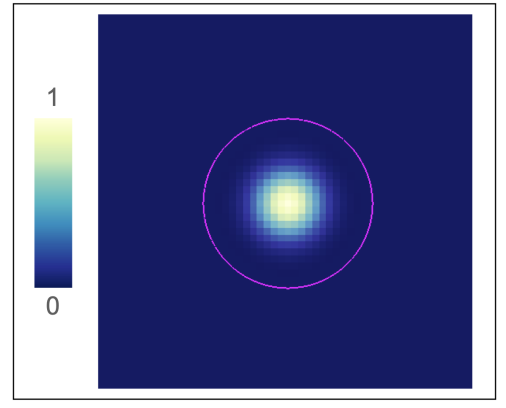

(a) $0 \mathrm{~s}$

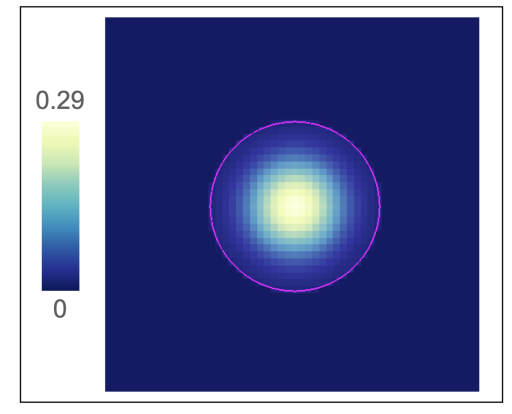

(b) $10 \mathrm{~s}$

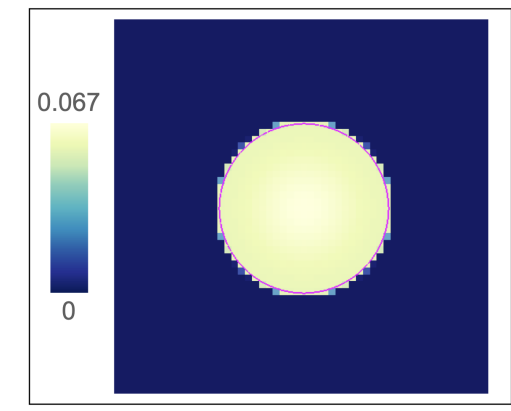

(c) $50 \mathrm{~s}$

Figure 3. Charge concentration $\left(\mathrm{C} / \mathrm{m}^{3}\right)$ in a static spherical droplet (pink) at different times as seen on a two-dimensional diametrical plane

The current formulation of PNP model is tested on a static spherical charged droplet in a cubic domain in the absence of a background electric field. The droplet initially contains a charge profile of the form

$$
c^{ \pm}=c_{i} \exp \left(r^{2} / 2 \eta^{2}\right)
$$

where $c_{i}$ is the maximum concentration, $r$ is the radial distance and $\eta$ governs the spatial decay of the concentration field. This diffusion problem of an initial concentration field in a spherical 


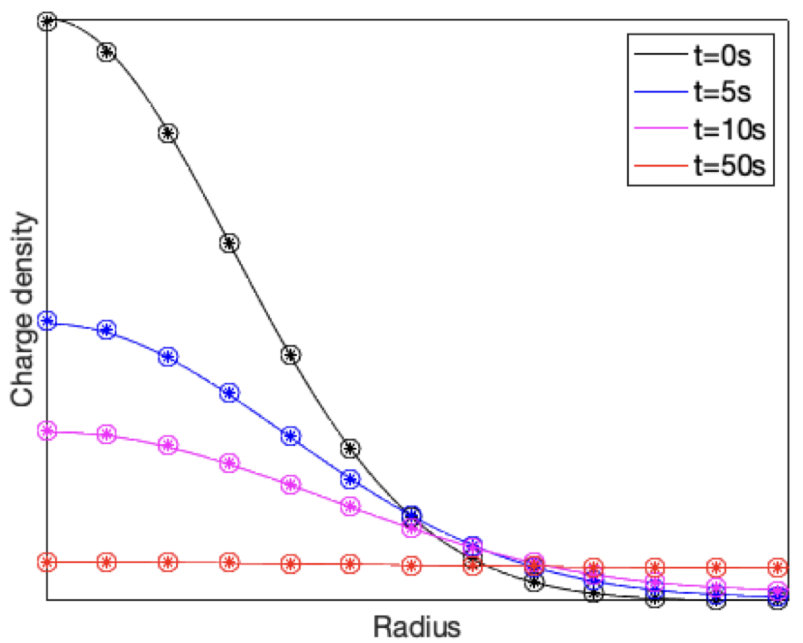

Figure 4. Solutions at different times for the charge diffusion test with the analytic solution shown with solid lines and numerically computed solution on a $61^{3}$ mesh shown with asterisks (cations) and circles (anions)

droplet with an impermeable surface has been described by [36] and has the solution

$$
c^{ \pm}(r, t)=\frac{3}{R^{3}} \int_{0}^{R} r^{2} f(r) \mathrm{d} r+\frac{2}{r R} \sum_{n=1}^{\infty} \exp \left(-D^{ \pm} \alpha_{n}^{2} t\right) \frac{\sin \left(\alpha_{n} r\right)}{\sin \left(\alpha_{n} R\right)} \int_{0}^{R} r^{\prime} f\left(r^{\prime}\right) \sin \left(\alpha_{n} r^{\prime}\right) \mathrm{d} r,
$$

where $R$ is the radius of the droplet, $\alpha_{n}$ s are the positive roots of $R \alpha_{n} \cot \left(R \alpha_{n}\right)=1$. The initial ion distribution for $c_{i}=1$ and $\eta=0.05$ is shown in Fig. 3a. Figs. 3b and 3c show the ions diffusing inside the droplet. Fig. 4 shows the temporal evolution of charge concentration computed on a $61^{3}$ mesh. Note that Eq. 8 is evaluated numerically using 200 terms of the infinite series and the integral is evaluated using the midpoint rule with 2000 intervals. Excellent agreement with the analytic solution is observed.

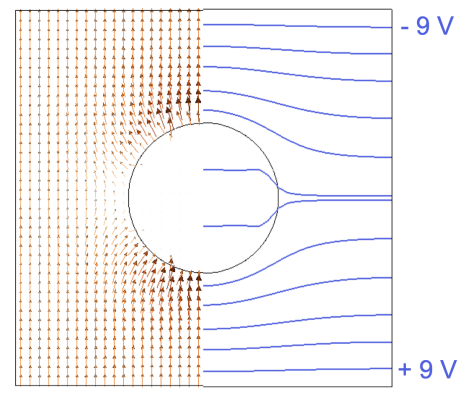

(a) Electric field lines (orange) and electric potential contour lines (purple)

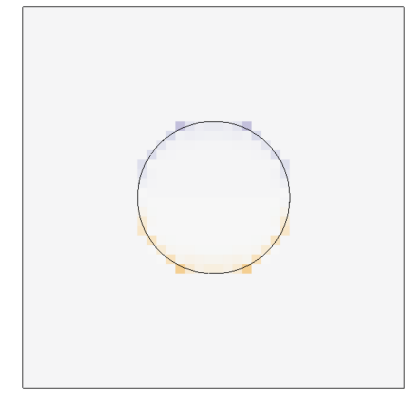

(b) Charge accumulation $\left(\mathrm{C} / \mathrm{m}^{3}\right)$ after $25 \mathrm{~s}$

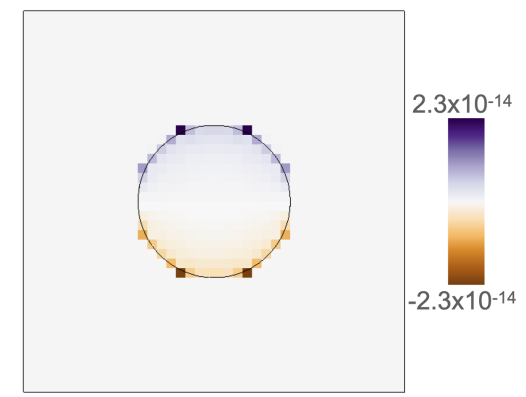

(c) Charge accumulation $\left(\mathrm{C} / \mathrm{m}^{3}\right)$ after $100 \mathrm{~s}$

Figure 5. Electromigration of ions in a static spherical droplet (black) at different times as seen on a two-dimensional diametrical plane

The formulation is also tested on a static spherical uniformly charged droplet in a cubic domain. The electric field is initialized as shown in Fig. 5a. It is to be noted that the electric field lines and electric potential contour bend near the interface due to the difference in permittivites between the two fluids phases. Despite a uniform initial charge distribution, the cations and anions move separately as dictated by the electric field. The positively charged anions "relax" by following the electric field towards the lower potential and vice versa. This results in charge separation, as seen in Fig. 5b. These charges eventually accumulate at the impermeable interface, i.e., cations accumulate close to the negatively charged boundary and anions near the positively 
charged boundary, seen in Fig. 5c. A higher charge concentration is seen in interfacial cells that contain lower liquid volumes because the charges accumulate in a smaller liquid volume.

\section{Conclusions and future work}

Experimental studies performed at the MCFL of the University of Washington show that an electrolyte jet, when injected through a grounded nozzle into a with a strong electric field, develops whipping instabilities and undergoes atomization. In this project, a PNP model has been developed to simulate multiphase electrolyte jet flows. Quantitative validation of the process of diffusion of ionic species has been performed. Electromigration has been observed qualitatively in a charged droplet in an electric field. The model developed will be used to understand the origination and propagation of whipping instabilities in electrolyte jets in an electric field. The numerical domain for simulating jets will be similar to that shown in Fig. 2 and uses e-Mesh that spans the entire domain and NS-Mesh spanning a narrow region along the propagation of the jet. Dimensions of NS-Mesh will be determined as more simulations are performed. Dirichlet BCs are set at the inflow region (grounded) and the top and bottom walls $(30 \mathrm{kV})$. All other regions on walls are set to Neumann BCs. Simulations are performed on multiple processors using message passing interface. Using this model, a mesh independence study will be performed to confirm convergent results, validate the model and to identify a reasonable mesh resolution to capture the physical processes. A parameter study will be performed in this numerical setup by varying electrolyte concentration, electric field strength and jet Reynolds number to understand the physical origin and the forces driving the propagation of these instabilities.

\section{Acknowledgements}

This material is based upon work supported by the National Science Foundation under Grant No. 1749779. We acknowledge the Multiphase \& Cardiovascular Flow Lab of the University of Washington for providing motivation and insight into the project.

\section{Nomenclature}

$\begin{array}{ll}\varepsilon & \text { Electric Permittivity } \\ \mathbf{E} & \text { Electric field vector } \\ \phi & \text { Electric potential } \\ D & \text { Diffusion constant } \\ c & \text { Ion concentration field } \\ \mathbf{N} & \text { lonic mass flux } \\ z & \text { Ionic valence } \\ k_{\mathrm{B}} & \text { Boltzmann constant } \\ e & \text { Elementary charge } \\ \mathrm{T} & \text { Ambient temperature }\end{array}$

\section{References}

[1] Garg, Koyal and Bowlin, Gary L. "Electrospinning jets and nanofibrous structures." Biomicrofluidics Vol. 5 No. 1 (2011): p. 013403.

[2] Reneker, Darrell H. and Yarin, Alexander L. "Electrospinning jets and polymer nanofibers." Polymer Vol. 49 No. 10 (2008): pp. 2387-2425.

[3] Onses, M Serdar, Sutanto, Erick, Ferreira, Placid M, Alleyne, Andrew G and Rogers, John A. "Mechanisms, capabilities, and applications of high-resolution electrohydrodynamic jet printing." Small Vol. 11 No. 34 (2015): pp. 4237-4266.

[4] Melcher, J. R. "Field-Coupled Surface Waves." Journal of Fluid Mechanics Vol. 229 No. 4 
(1963): pp. 764-764.

[5] Saville, D. A. "Stability of Electrically Charged Viscous Cylinders." The Physics of Fluids Vol. 14 No. 6 (1971): pp. 1095-1099.

[6] Huebner, A. L. and Chu, H. N. "Instability and breakup of charged liquid jets." Journal of Fluid Mechanics Vol. 49 No. 2 (1971): p. 361-372.

[7] Saville, D. A. "Electrohydrodynamic Stability: Fluid Cylinders in Longitudinal Electric Fields." The Physics of Fluids Vol. 13 No. 12 (1970): pp. 2987-2994.

[8] Saville, D. A. "Electrohydrodynamic stability: effects of charge relaxation at the interface of a liquid jet." Journal of Fluid Mechanics Vol. 48 No. 4 (1971): p. 815-827.

[9] González, Heliodoro, García, F. Javier and Castellanos, Antonio. "Stability analysis of conducting jets under ac radial electric fields for arbitrary viscosity." Physics of Fluids Vol. 15 No. 2 (2003): pp. 395-407.

[10] Guerrero, Josefa, Rivero, Javier, Gundabala, Venkata R., Perez-Saborid, Miguel and Fernandez-Nieves, Alberto. "Whipping of electrified liquid jets." Proceedings of the National Academy of Sciences of the United States of America Vol. 111 No. 38 (2014): pp. 13763-13767.

[11] Shin, Y. M., Hohman, M. M., Brenner, M. P. and Rutledge, G. C. "Electrospinning: A whipping fluid jet generates submicron polymer fibers." Applied Physics Letters Vol. 78 No. 8 (2001): pp. 1149-1151.

[12] Riboux, Guillaume, Marnín., Alvaro G., Loscertales, Ignacio G. and Barrero, Antonio. "Whipping instability characterization of an electrified visco-capillary jet." Journal of Fluid Mechanics Vol. 671 (2011): pp. 226-253.

[13] Arne, Walter, Marheineke, N., Pérez-Saborid, M., Rivero-Rodríguez, J., Wegener, R. and Wieland, M. "Whipping of Electrified Visco-Capillary Jets in Airflows." SIAM J. Appl. Math. Vol. 78 (2018): pp. 343-371.

[14] Fridrikh, Sergey V., Yu, Jian H., Brenner, Michael P. and Rutledge, Gregory C. "Nonlinear Whipping Behavior of Electrified Fluid Jets." Polymeric Nanofibers. Vol. 918 of ACS Symposium Series. American Chemical Society (2006): pp. 4-36.

[15] Fridrikh, Sergey V., Yu, Jian H., Brenner, Michael P. and Rutledge, Gregory C. "Controlling the Fiber Diameter during Electrospinning." Phys. Rev. Lett. Vol. 90 (2003): p. 144502.

[16] Horng, Tzyy-Leng, Lin, Tai-Chia, Liu, Chun and Eisenberg, Bob. "PNP equations with steric effects: a model of ion flow through channels." The Journal of Physical Chemistry $B$ Vol. 116 No. 37 (2012): pp. 11422-11441.

[17] de Paula, Jamile Lorena, da Cruz, José Adauto, Lenzi, Ervin Kaminski and Evangelista, Luiz Roberto. "Immittance response of an electrolytic cell in the presence of adsorption, generation, and recombination of ions." Journal of Electroanalytical Chemistry Vol. 682 (2012): pp. 116-120.

[18] Choi, Yong Seok and Kim, Sung Jin. "Electrokinetic flow-induced currents in silica nanofluidic channels." Journal of colloid and interface science Vol. 333 No. 2 (2009): pp. 672-678.

[19] Moya, AA, Hayas, A and Horno, J. "Steady-state, transient and small-amplitude AC responses of an electrochemical cell with immobile background charge:: A network approach." Solid state ionics Vol. 130 No. 1-2 (2000): pp. 9-17.

[20] Guillaume, Blanquart, Perrine, Pepiot-Desjardins and Heinz, Pitsch. "Chemical mechanism for high temperature combustion of engine relevant fuels with emphasis on soot precursors." Combustion and Flame Vol. 156 (2009): pp. 588-607.

[21] Owkes, Mark and Desjardins, Olivier. "A mass and momentum conserving unsplit semiLagrangian framework for simulating multiphase flows." Journal of Computational Physics Vol. 332 (2017): pp. 21-46.

[22] Desjardins, Olivier, Blanquart, Guillaume, Balarac, Guillaume and Pitsch, Heinz. "High order conservative finite difference scheme for variable density low Mach number turbulent flows." Journal of Computational Physics Vol. 227 (2008): pp. 7125-7159. 
[23] Desjardins, Olivier, Moureau, Vincent and Pitsch, Heinz. "An accurate conservative level set/ghost fluid method for simulating turbulent atomization." Journal of Computational Physics Vol. 227 (2008): pp. 8395-8416.

[24] Owkes, Mark and Desjardins, Olivier. "A mesh-decoupled height function method for computing interface curvature." Journal of Computational Physics Vol. 281 (2015): pp. 285300.

[25] Poppel, B. P. Van, Desjardins, O. and Daily, J. W. "A ghost fluid, level set methodology for simulating multiphase electrohydrodynamic flows with application to liquid fuel injection." Journal of Computational Physics Vol. 229 (2010): pp. 7977-7996.

[26] Van Poppel, Bret P. "Numerical methods for simulating multiphase electrohydrodynamic flows with application to liquid fuel injection." Ph.D. Thesis, West Point ETD. 2010.

[27] Sheehy, Patrick and Owkes, Mark. "Numerical study of electric Reynolds number on electrohydrodynamic assisted atomization." Atomization and Sprays Vol. 27 No. 7 (2017): pp. 645-664.

[28] Saville, D. A. "Electrohydrodynamics: The Taylor-Melcher Leaky Dielectric Model." Annual Review of Fluid Mechanics Vol. 29 No. 1 (1997): pp. 27-64.

[29] Nernst, Walther. "Die elektromotorische wirksamkeit der jonen." Zeitschrift für physikalische Chemie Vol. 4 No. 1 (1889): pp. 129-181.

[30] Planck, Max. "Ueber die erregung von electricität und wärme in electrolyten." Annalen der Physik Vol. 275 No. 2 (1890): pp. 161-186.

[31] Prohl, Andreas and Schmuck, Markus. "Convergent finite element discretizations of the Navier-Stokes-Nernst-Planck-Poisson system." ESAIM: Mathematical Modelling and Numerical Analysis - Modélisation Mathématique et Analyse Numérique Vol. 44 No. 3 (2010): pp. 531-571.

[32] Frank, F., Ray, N. and Knabner, P. "Numerical investigation of homogenized Stokes-Nernst-Planck-Poisson systems." Computing and Visualization in Science Vol. 14 (2011): p. 385-400.

[33] Pham, Van Sang, Li, Zirui, Lim, Kian Meng, White, Jacob K. and Han, Jongyoon. "Direct numerical simulation of electroconvective instability and hysteretic current-voltage response of a permselective membrane." Phys. Rev. E Vol. 86 (2012): p. 046310. .

[34] Druzgalski, C. L., Andersen, M. B. and Mani, A. "Direct numerical simulation of electroconvective instability and hydrodynamic chaos near an ion-selective surface." Physics of Fluids Vol. 25 No. 11 (2013): p. 110804.

[35] Kourmatzis, Agisilaos and Shrimpton, John S. "Electrohydrodynamics and charge injection atomizers: A review of the governing equations and turbulence." Atomization and Sprays Vol. 19 No. 11 (2009): pp. 1045-1063.

[36] Crank, John. The mathematics of diffusion. Oxford university press (1979). 